\title{
CivaSheet Directional Pd103 Brachytherapy Source
}

National Cancer Institute

\section{Source}

National Cancer Institute. CivaSheet Directional Pd103 Brachytherapy Source. NCI

Thesaurus. Code C138130.

A proprietary brachytherapy source designed to deliver a radiation dose in a single direction. The device incorporates Palladium-103 as a radiation source and utilizes gold shielding to deliver the radiation dose in a unidirectional, planar manner. CivaSheet is flexible, bioabsorbable and features a polymer-encapsulated radiation source. 